COMMENT. While MRI data may not be reliable in localizing an epileptogenic area, measurements of brain volume reduction in partial epilepsies may prove of value in prognosis, especially in potential surgical cases. The correlation of IQ with brain volume in the above MRI study of children with epilepsy corroborates similar findings in normal children and adolescents at Johns Hopkins University (Reiss AL et al. Brain 1996;119:1763; and Progress in Pediatric Neurology III, 1997;p294).

Mitochondrial complex I deficiency is reported in the hippocampal epileptic focus of patients with temporal lobe epilepsy. (Kunz WS et al. Ann Neurol November 2000;48:766-773). Mitochondrial dysfunction is a possible mechanism involved in the neuronal excitability in temporal lobe epilepsy.

\title{
USE AND ABUSE OF THE ELECTROENCEPHALOGRAM
}

The utility of the electroencephalogram (EEG) to evaluate clinical situations in epilepsy is reviewed from the Department of Clinical Neurophysiology, King's College Hospital, London, UK. Based on an analysis of articles available in Medline, Cochrane, and the Internet, and personal experience, the utility of the EEG is not satisfactorily addressed. A referral for an EEG should contain a clear statement of the clinical problem and the reasons for obtaining an EEG. Lack of communication between the clinician and the neurophysiologist may result in abuse of the EEG and minimize its usefulness. The diagnosis of epilepsy is made essentially on clinical evidence, and a referral for a routine EEG for this purpose is usually an abuse. In patients with epilepsy, the first EEG will confirm the diagnosis in $80 \%$. Epileptiform activity in the EEG considerably enhances the likelihood of epilepsy. It may also answer a frequent question "Is it epilepsy?" Video EEG telemetry or ambulatory EEG may be required to distinguish epilepsy vs nonepileptic attack disorder, or absence vs daydreaming. The EEG may be used to distinguish a seizure type or syndrome and its associated clinical prognosis; it can rarely determine etiology. It may provide the first localizing evidence for a lesion, and its utility in presurgical evaluation of epilepsy has not been entirely displaced by imaging techniques. In children, the EEG can help to determine when it is safe or appropriate to discontinue antiepileptic drugs. Interpretation of the EEG requires detailed information regarding the clinical situation, and close liaison between the referring clinician and the EEG department. (Fowle AJ, Binnie CD. Uses and abuses of the EEG in epilepsy. Epilepsia 2000;41(Suppl 3):S10-S18). (Reprints: Dr Adrian J Fowle, Department of Clinical Neurophysiology, King's College Hospital, Denmark Hill, London SE5 9RS, UK).

COMMENT. The authors conclude that the EEG has many uses in the evaluation of epilepsy, but attention to detail is essential in the referral request. Our own Epilepsy Center at Children's Memorial Hospital, Chicago, directed by Drs Nordli, Stack, and Kelly, requires a detailed referral form to be completed, when requesting an EEG. If this practice was more generally adopted, the utility of the EEG in the management of epilepsy would be increased.

EEG in evaluation of the first nonfebrile seizure is addressed in a recent report of the quality standards subcommittee of the American Academy of Neurology, Child Neurology Society, and American Epilepsy Society (Hirtz D, Ashwal S, Berg A et al. Neurology Sept (1 of 2) 2000;55:616-623). (Reprints: QSS, American Academy of Neurology, 1080 Montreal Ave, St Paul, MN 55116). Based on a review of available evidence in the literature, routine EEG was recommended as part of the diagnostic evaluation of the first nonfebrile seizure in children. The 
authors disagreed with the conclusion of Gilbert and Buncher (Neurology 2000;54:635-641) that the EEG should not be routinely performed after a first seizure because it does not alter treatment. In contrast, the committee concludes that the EEG helps in determination of seizure type, the diagnosis of epilepsy syndromes, and risk of recurrence; it provides information on long-term prognosis; it influences the decision to perform neuroimaging studies; and it may affect further management. The optimal timing of the EEG is not clear. It is most likely to show abnormalities when obtained within 24 hours of the seizure, although postictal slowing may be transient and must be interpreted with caution.

\section{BENIGN CHILDHOOD OCCIPITAL EPILEPSY}

The clinical and EEG features of the syndrome of benign childhood occipital epilepsy (Panayiotopoulos syndrome [PS]) were studied in 66 patients seen between 1990 and 1997 at the Hospital Nacional de Pediatria "Juan P Garrahan," Buenos Aires, Argentina. In the same time period, 145 children were diagnosed with the syndrome of benign childhood epilepsy with centrotemporal spikes (BECTS). Inclusion criteria for PS included ictal vomiting (100\%), deviation of eyes and head (98\%), clonic partial seizures (33\%), impaired consciousness, and secondary generalization to convulsions (39\%). Prolonged seizures and partial status epilepticus occurred in one third. Seizures were nocturnal in all patients and also diurnal in one third. Ictal visual symptoms occurred in 9\%. Five had concurrent rolandic epilepsy (BECTS) and another 5 developed rolandic seizures after remission of PS. Prognosis was excellent. A single seizure occurred in 30\%, $49 \%$ had infrequent sporadic recurrences, and $18 \%$ had seizures every 1 to 5 months. The EEG showed occipital spikes in all 66 children; spikes were bilateral or unilateral, activated by sleep, and paroxysms were blocked by eye-opening. One-third also had centrotemporal and frontal spikes. None had generalized spike-wave discharges. Following treatment with antiepileptic drugs, principally carbamazepine, $90 \%$ had no further seizures. Seizures remitted within 1 to 8 years (mean 5.5 years) from onset despite persistent EEG abnormalities in $75 \%$. (Caraballo R, Cersosimo R, Medina C, Fejerman N. Panayiotopoulos-type benign childhood occipital epilepsy. Neurology October (2 of 2) 2000;55:1096-1100).

(Reprints: Dr Robert Caraballo MD, Combate de los Pozos 1881 (1245), Buenos Aires, Argentina).

COMMENT: The authors conclude that Panayiotopoulos syndrome (PS), or Ptype childhood epilepsy with occipital paroxysms (CEOP), is a relatively common, homogeneous, and benign syndrome that deserves wider recognition. Unusual seizure manifestations such as vomiting and a first prolonged seizure may mimic encephalitis or intoxication. Most reports have emphasized the excellent prognosis, with remission in 1 to 2 years, and one-third of cases with only one seizure.

An editorial by Berg AT and Panayiotopoulos CP (Neurology Oct (2 of 2) 2000;55:1073-1074) outlines the typical presenting symptoms of the syndrome, and proposes a continuum of interrelated, benign, childhood, partial epilepsy syndromes. PS prevalence is about half that of BECTS. They emphasize the frequent initial presentation with status epilepticus and vomiting, symptoms that may suggest a more serious underlying process, leading to misdiagnosis. They advocate inclusion of the PS syndrome in the ILAE classification of epileptic syndromes.

Interictal clonic/atonic symptoms occurring in BECTS may be caused by overmedication and may warrant AED withdrawal or substitution (de Saint-Martin A et al. Neurology Oct (2 of 2) 2000;55:1241-1242). 